\title{
Acción tóxica del tetratiomolibdato de amonio sobre el tejido testicular en ratas*
}

\author{
Trulls, H.E. .; Brem, J.J.; Sánchez Negrette, M.²; Pochon, D.O. ${ }^{3}$; Ortíz, M.L. ${ }^{1}$ \\ ${ }^{1}$ Cátedra de Biofísica, ${ }^{2}$ Patología General y Sistemática, ${ }^{3}$ Bioestadística, Facultad de Ciencias Veterinarias, \\ UNNE, Sargento Cabral 2139, Corrientes (3400), Argentina. Tel/Fax (0378-430101-Int.150), \\ E-mail: htrulls@vet.unne.edu.ar.
}

\begin{abstract}
Resumen
Trulls, H.E.; Brem, J.J.; Sánchez Negrette, M.; Pochon, D.O.; Ortíz, M.L.: Acción tóxica del tetratiomolibdato de amonio sobre el tejido testicular en ratas. Rev. vet. 19: 2, 125-129, 2008. La acción tóxica del molibdeno, por sí mismo o por su interacción con el metabolismo del cobre, podría ocasionar trastornos sobre diversos tejidos. Con el fin de provocar una deficiencia secundaria de cobre, se procedió al agregado de tetratiomolibdato de amonio (TTMo) en la dieta de ratas, para evaluar sus efectos sobre la función reproductiva. En rumiantes, la toxicidad del Mo es provocada por la formación de tetratiomolibdatos por la microflora ruminal. Con este producto sintetizado in vitro, se pudo reproducir la enfermedad en ratas que, como el resto de los monogástricos, son particularmente resistentes a la intoxicación natural del Mo por carecer del ambiente digestivo adecuado para dicha transformación. Para lograr el objetivo propuesto, se valoró la respuesta de la testosterona tras el estímulo de gonadorrelinas $(\mathrm{GnRH})$ y mediante estudios anatomohistopatológicos se determinó la integridad estructural del tejido testicular. Se utilizaron 40 ratas de sexo macho de la cepa Wistar, de 3 meses de edad; los grupos tratados recibieron durante 120 días dosis de $3 \mathrm{mg}$ de TTMo/100 $\mathrm{ml}$ de agua de bebida. En este ensayo de molibdenosis experimental se logró una sintomatología clínica y bioquímica compatible con una deficiencia secundaria de cobre. Las diferencias entre grupos tratados y testigos en la respuesta de testosterona después de la inyección de GnRH, fueron significativas, indicando una función endocrina comprometida. Los ejemplares tratados revelaron disminución del tamaño testicular, túbulos seminíferos con fenómenos degenerativos del epitelio germinal, necrosis y desaparición de espermátides y espermatozoides. Algunos testículos presentaban únicamente espermatogonias y células de Sertoli con fenómenos de vacuolización citoplasmática, picnosis nuclear y células gigantes fagocíticas multinucleadas. Las lesiones en el tejido intersticial se caracterizaron por fibrosis leve y depleción de células intersticiales de Leydig.
\end{abstract}

Palabras clave: rata, molibdenosis, deficiencia de cobre, testosterona, daño testicular.

\begin{abstract}
Trulls, H.E.; Brem, J.J.; Sánchez Negrette, M.; Pochon, D.O.; Ortíz, M.L.: Toxic action of ammonium tetrathiomolybdate on testicular tissue, in rats. Rev. vet. 19: 2, 125-129, 2008. Molybdenum toxicity may cause disturbances on several tissues either by itself or through its interaction with copper metabolism. To develop a secondary copper deficiency, tetrathiomolybdenum (TTMo) was supplemented to male rats to evaluate its effects on reproductive performance. In ruminants, Mo toxic action is due to the production of tetrathiomolybdates by ruminal microflora. With this in vitro synthesized metabolite, it was possible to reproduce this ruminant disease syndrome in rats, a monogastric species that is particularly resistant to the natural intoxication of Mo, considering the inappropriate digestive environment for such transformation. The objective of this work was to measure testosterone response after gonadorreline (GnRH) injections, as well as to perform anatomopathologic studies to determine the structural integrity of testicular tissues. Forty male 3 months-old Wistar rats, were used. Treated group received daily doses of $3 \mathrm{mg}$ of TTMo/100ml in drinking water during 120 days. In this experimental molybdenosis assay, clinical and biochemical symptomatologies were correlated with a secondary deficiency of copper. The differences between treated and control groups regarding to testosterone response after $\mathrm{GnRH}$ injection were significant, showing an abnormal endocrine function. Morphological studies of other two lots showed a decrease in testicular size of treated animals, and seminiferous tubules with degenerative
\end{abstract}

Recibido: 24 setiembre 2008 / Aceptado: 6 noviembre 2008.

*Este trabajo es parte de la tesis doctoral del MV Horacio Eduardo Trulls, realizada en la Facultad de Ciencias Veterinarias-UNNE. 


\begin{abstract}
phenomena of the germinal epithelium, necrosis and spermatozoid absence. Some testicles presented only spermatogonia and/or Sertoli cells with citoplasmatic vacuolation, nuclear picnosis and multinucleated phagocytic gigant cells. Lesions of the interstitial tissue were mild fibrosis and depletion of Leydig interstitial cells.
\end{abstract}

Key words: rat, molybdenosis, copper deficiency, testosterone, testicular damage.

\section{INTRODUCCIÓN}

Altos niveles de molibdeno dietario en rumiantes pueden ocasionar, por sí mismos o a través de su interacción sobre el metabolismo del cobre, diversos trastornos, entre ellos reproductivos, tanto en machos como en hembras ${ }^{2,3,9,10,13,14}$. La molibdenosis es también conocida con el nombre de hipocuprosis secundaria, deficiencia secundaria de cobre o deficiencia condicionada de cobre. Afecta la producción ganadera en grandes regiones de Argentina y otras partes del mundo. Diversos trastornos de la reproducción han sido asociados con deficiencias primaria y secundaria de cobre en bovinos. La suplementación con $\mathrm{Cu}$ ha dado resultados muy dispares; en algunos casos fueron exitosos ${ }^{6,17} \mathrm{y}$ en otros no se pudieron observar respuestas favorables 16,19 .

Por otro lado, desequilibrios hormonales del eje hipotalámico-hipofisario-gonadal han sido imputados al efecto per se del molibdeno, antes que a la disminución de la reserva orgánica de cobre ${ }^{1,15}$. La libido del macho depende del soporte endocrino dado por la testosterona secretada por las células intersticiales de Leydig por acción de LH hipofisiaria, en respuesta al factor liberador de LH hipotalámico (GnRH). En 1951 se observaron lesiones testiculares en toros de raza Holstein sometidos a dosis altas de molibdeno ${ }^{18}$. Estudios con tetratiomolibdato (TTMo) realizados en ratas demostraron que esta especie resultó ser un modelo experimental adecuado de la molibdenosis en monogástricos, ideal para trabajar en condiciones mejor controladas respecto a las especies mayores ${ }^{4,7}$.

En el presente ensayo se propuso valorar el nivel de testosterona tras el estímulo de GnRH, así como evaluar la integridad estructural del testículo de ratas sometidas a una dieta complementada con TTMo.

\section{MATERIAL Y MÉTODOS}

Para evaluar los efectos tóxicos generales del TTMo sobre los animales, se consideraron como parámetros de referencia aquellos que resultaron de utilidad en otros trabajos ${ }^{4,5}$, como ser sintomatología clínica (diarrea y pérdida del estado nutricional), evolución del peso corporal (dos veces por semana), determinación del hematocrito (presencia o no de anemia) y niveles de cobre plasmático (hipocupremia) hacia el final de la prueba.

Síntesis de TTMo. El tetratiomolibdato de amonio utilizado fue obtenido in vitro a partir de heptamolibdato de amonio disuelto en amoníaco, burbujeado con
$\mathrm{H}_{2} \mathrm{~S}$ proveniente de un aparato de Kipp por acción del $\mathrm{HCl}$ diluido sobre piedras de sulfuro férrico (piritas). El producto sintetizado fue caracterizado mediante absorción espectral en UV/visible con un espectrofotómetro con módulo digital computarizado marca Perkin Elmer modelo Lambda 25. El seguimiento de las densidades ópticas se realizó desde $230 \mathrm{~nm}$ hasta $510 \mathrm{~nm}$, con intervalos de $5 \mathrm{~nm}$, comprobándose el grado de pureza mediante los 3 picos característicos de máxima absorbancia obtenidos (240, 320 y $470 \mathrm{~nm}$ ).

Alimentación y tratamientos. Los animales fueron alimentados con un balanceado comercial adecuado para la especie. Determinaciones químicas realizadas en el alimento mediante un espectrómetro de absorción atómica GBC Modelo 932-AB, arrojaron los siguientes valores: hierro $33 \mathrm{ppm}$, cobre 5,1 ppm y molibdeno $0,40 \mathrm{ppm}$. Para provocar la deficiencia secundaria de cobre, además de la dieta, se le suministró TTMo en dosis de $3 \mathrm{mg} / 100 \mathrm{ml}$ en el agua de bebida, preparada diariamente y con consumo controlado (que alcanzó un promedio de $25 \mathrm{ml} / \mathrm{animal} / \mathrm{día})$. Al relacionar este promedio con el análisis químico del alimento se deduce que el lote tratado fue sometido a una dieta con una relación Cu:Mo de 1:3 en tanto que en los testigos fue de 6:1.

Animales y muestreos. Se utilizaron 40 ratas de sexo macho de la cepa Wistar, distribuidas de la siguiente manera: a) ensayo para evaluar respuesta de testosterona - grupo tratado y grupo testigo $\mathrm{n}=11$ cada uno- y b) para estudios anatomohistopatológicos -grupo tratado $\mathrm{n}=10$ y grupo testigo $\mathrm{n}=8-$. El consumo de dieta y bebida fue ad libitum y el control de peso se efectuó dos veces por semana. Las pruebas fueron iniciadas con animales de 3 meses de edad y con pesos corporales homogéneos $(200 \pm 10$ g). Hacia el día 120 de la prueba se extrajeron muestras de sangre por vía intracardíaca, anticoaguladas con EDTA; simultáneamente se le inyectaron $0,2 \mathrm{ml}$ de GnRH (Receptal ${ }^{\mathrm{R}}$, Hoechst Roussel Vet) por vía i.m.; transcurridas 2 horas se les extrajo nuevamente sangre. Ambas muestras se utilizaron para determinar los valores basales y de respuesta de testosterona tras el estímulo de gonadorrelinas.

Estudio anatomohistopatológico. Se trabajó con testículos de grupos tratados y testigos, fijados y procesados según técnicas clásicas para bloques parafinados, cortados a un espesor de $5 \mu \mathrm{m}$ y coloreados con hematoxilina-eosina (HyE). Los animales fueron sacrificados hacia los 4 meses de tratamiento, previa anestesia 
con éter y sangrados a blanco para las determinaciones de hematocrito y cupremia.

Determinaciones químicas. La concentración sanguínea de cobre se efectuó por espectrometría mediante el método de la batocuproína, previa desproteinización del suero con ácido tricloroacético, lectura a $480 \mathrm{~nm}$. El valor de hematocrito fue determinado según técnica del microhematocrito con centrifugado a $15300 \mathrm{~g}$ durante 5 minutos y lectura en ábaco. Los niveles de testosterona se evaluaron por radioinmunoanálisis (RIA) con hormona marcada con ${ }^{125}$ Iodo de alta actividad específica, según técnica del doble anticuerpo unido a partículas de hierro, con separación magnética de las fracciones libre y unida, reactivos de Laboratorios Serono. La medición de actividad radiactiva gamma fue realizada en espectrómetro digital marca Alfa Nuclear Modelo ZX, con contador de pozo con cristal de yoduro de sodio activado con talio.

Análisis estadístico. Las variables peso corporal, hematocrito y cupremia (cuantitativas continuas) fueron tratadas mediante estadísticas descriptivas, intervalos de confianza de $95 \%$ y test " $t$ " de Student. Todas las variables paramétricas cumplieron los supuestos básicos de normalidad y homogeneidad de la variancia. La diferencia entre tratamientos se obtuvo mediante análisis de varianza (ANOVA) para un diseño completamente aleatorio de una sola vía, previa constatación de la distribución normal de la población mediante la prueba de Shaphiro-Wilk; la homogeneidad de la varianza se verificó mediante la prueba de Bartlett. Para comparar el efecto de la hormona en cada grupo (testigo y tratado), previo y a las 2 horas post-inyección de GnRH, se aplicó una prueba de " $t$ " apareada. Para medir si los incrementos de la hormona fueron diferentes entre ambos grupos, al no cumplirse el supuesto de independencia, los datos fueron tratados mediante un análisis de la varianza para medidas repetidas.

\section{RESULTADOS}

Los grupos sometidos a un exceso de molibdeno manifestaron pérdidas del estado nutricional y frecuentes diarreas a partir de los 7 a 10 días del ensayo. En la Tabla 1 se observa que el grupo tratado, respecto al grupo testigo, registró disminución del peso corporal y descensos importantes de hematocrito y cupremia al final de la prueba. El análisis de la varianza arrojó valores de peso corporal, hematocrito y cupremia con diferencias muy significativas entre tratamientos $(p<0,001)$, siendo las diferencias entre medias de 33,3 g, 10\% y $48,2 \mathrm{ug} / \mathrm{dl}$, respectivamente.
La Tabla 2 muestra que inicialmente no existieron diferencias significativas entre los niveles de testosterona de ambos grupos. Sin embargo, a las 2 horas de la inyección de GnRH se registraron diferencias significativas $(\mathrm{p}<0,01)$ de las concentraciones hormonales entre testigos y tratados. Así, las diferencias entre medias finales versus iniciales fueron de $0,93 \mathrm{ng} / \mathrm{ml}$ en testigos $\mathrm{y}$ de $0,65 \mathrm{ng} / \mathrm{ml}$ en tratados.

El examen macroscópico de los testículos permitió observar disminución del tamaño en los animales tratados respecto a los testigos. A la microscopía (Figuras 1 y 2), en los tratados se observaron túbulos seminíferos con severos fenómenos degenerativos caracterizados por encogimiento y colapso tubular, junto a un adelgazamiento del epitelio germinal, vacuolización citoplasmática, descamación de células germinales, picnólisis de los núcleos de los espermatocitos y formación de células gigantes fagocíticas mononucleadas y multinucleadas. En ocasiones, los túbulos presentaron únicamente espermatogonias y células de Sertoli. En la mayoría de los testículos estudiados no se observaron espermátides ni espermatozoides. Las lesiones en el tejido intersticial se caracterizaron por fibrosis leve y depleción de células de Leydig.

\section{DISCUSIÓN}

Los valores obtenidos de peso corporal, hematocrito y cupremia, demuestran el comportamiento esperado en el modelo experimental bajo las condiciones del ensayo, tal como fuera comunicado en otros trabajos ${ }^{4,5}$.

La menor respuesta de testosterona al estímulo de GnRH en los animales tratados con molibdeno podría deberse a una disminución del cobre tisular, tal como fuera observado en estudios realizados sobre el contenido de este mineral en diversos órganos de ratas, en 
los que se demostró la influencia de la edad y del sexo en su distribución, dependiendo de las influencias hormonales por reducción en la actividad de enzimas $\mathrm{Cu}-$ dependientes ${ }^{8,20}$. Asimismo, ratas gonadectomizadas y tratadas previamente con esteroides y con un complejo $\mathrm{Cu}-\mathrm{LHRH}$, mostraron una mayor liberación de LH y FSH ${ }^{11}$. Si bien la evaluación en la respuesta de testosterona tras estímulo de GnRH es una prueba que depende de la liberación previa de LH, se puede inferir, sin llegar a descartar lo que pudo haber ocurrido a nivel de hipófisis por no haberse podido determinar niveles de LH, que la función endocrina testicular estuvo comprometida a juzgar por los daños tisulares encontrados a este nivel.

Las lesiones histopatológicas observadas en la mayoría de los testículos estudiados, como ausencia de espermátides y espermatozoides, lesiones de fibrosis

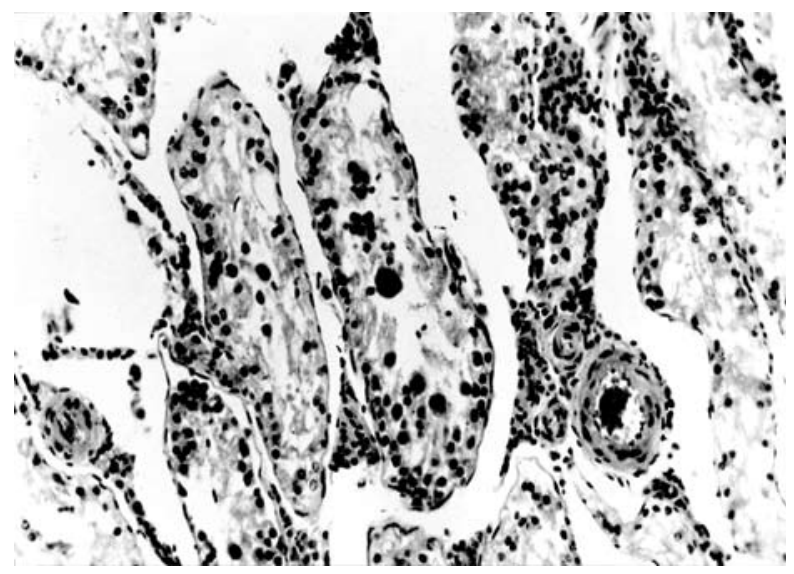

Figura 1. Testículo de animal tratado. Túbulos seminíferos atrofiados, con fenómenos degenerativos caracterizados por pérdida de epitelio germinal, necrosis y presencia de células gigantes. El intersticio presenta escasas células de Leydig (H y E, 200X).

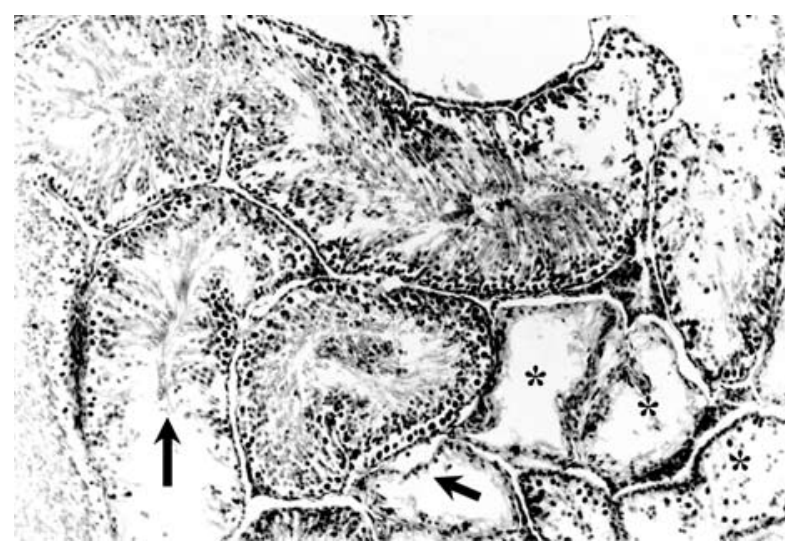

Figura 2. Testículo de animal tratado. Túbulos seminíferos (en el centro y a la izquierda de la imagen) con fenómenos degenerativos moderados, presencia de espermatogonias, células de Sertoli y algunos espermatocitos primarios (flechas). Hacia la derecha de la imagen (asteriscos) se observa un área con túbulos seminíferos encogidos, con pérdida total del epitelio germinal (H y E, 100X). y disminución de células intersticiales de Leydig, son similares a las observadas en toros de raza Holstein que presentaban ausencia de libido al ser sometidos a dosis altas de molibdeno ${ }^{18} \mathrm{y}$ a las observadas en testículos de ratas por acción del molibdato de sodio ${ }^{12}$.

En conclusión, el modelo propuesto de molibdenosis experimental fue idóneo y se logró una sintomatología clínica y bioquímica compatible con deficiencia secundaria de cobre. Si bien hubo respuesta de testosterona al estímulo de gonadorrelinas en ambos grupos, en el tratado fue significativamente menor. Asimismo pudo corroborarse la presencia de lesiones testiculares importantes poniendo en evidencia una morfofisiología testicular comprometida por la acción de la sal de molibdeno utilizada.

Agradecimientos: A la Lic. María Emilia García Denegri, por elaborar el abstract. A la SGCYT-UNNE por apoyar económicamente el estudio.

\section{REFERENCIAS}

1. Auza N, Acuña C, Casaro JP, Braun JP. 1983. Effects de l'administration de diverses preparations chez de bovis carences en cuivre. Biologie Prospective. $5^{\circ}$ Colloque International de Pont-a-Mousson (Francia), Ed. Masson, p. $1145-1147$.

2. Auza N. 1983. Le cuivre chez les ruminants. Une revieu. Ann Rech Vet 14: 21-37.

3. Brem JJ, Roux JP. 1991. Aspectos clínico-hematológicos de la molibdenosis experimental en bovinos con y sin tratamiento parenteral de cobre. Vet Arg 8: 38-49.

4. Brem JJ, Pochon DO, Trulls HE. 1997. Deficiencia condicionada de cobre en ratas provocada por un exceso de molibdeno. Anales XVII Jornadas Interdiciplinarias de Toxicología (Buenos Aires), p. 37.

5. Brem JJ, Pochon DO, Trulls HE. 2000. Toxicidad del heptamolibdato y del tetratiomolibdato de amonio en ratas. Rev Vet 10/11: 3-6.

6. Brem JJ, Mestre J, Pochon DO, Trulls HE. 2002. Alteraciones del ciclo estral provocadas por un alto ingreso de molibdeno en vaquillonas Brangus y respuesta a la suplementación con cobre. Rev Vet 12/13: 28-33.

7. Brem JJ. 2005. Hystopatological injuries of the bone tissue in rats treated with high dose of ammonium tetrathiomolibdate. Acta Tox Arg 13: 119.

8. Bremner I, Williams RB, Young BW. 1981. The effects of age, sex and zinc status on the accumulation of (copperzinc)-metallothionein in rat kidneys. J Inorg Biochem 14 : 135-146.

9. Farina JN, Farina JL, Farina CM. 1983. El desbalance mineral como causa de bajos porcentajes de preñez. Gac Vet 45: 653-656.

10. Hunter AP. 1977. Some nutritional factors affecting the fertility of dairy cattle. $N Z$ Vet $J$ 25: 305-309.

11. Kochman K, Gajewska A, Kozlowski H, Masiukiewicz E, Rzeszotarska B. 1992. Increased LH and FSH release from the anterior pituitary of ovariectomized rat in vivo, 
by copper, nickel and zinc-LHRH complexes. J Inorg Biochem 48: 41-46.

12. Pandey R, Singh SP. 2002. Effects of molibdenum on fertility of male rats. Biomedicals 13: 65-72.

13. Phillippo M, Humphries WR, Laurence CB, Price J. 1982. Investigation of the effect of copper status and therapy on fertility in beef suckler herds. J Agric Sci 99: 359-364.

14. Phillippo M, Humphries WR, Atkinson T. 1985. The effect of molybdenum on fertility in the cow. Proc Nutr Soc 44: 82 .

15. Phillippo M, Humphries WR, Garthwaite PP. 1987. The effect of dietary molybdenum and iron on copper status and growth in cattle. J Agric Sci 109: 315-320.
16. Poole DB, Walshe MJ. 1970. Trace elements metabolism in animals, Ed. Mills, Edinburgh, p. 448.

17. Sastry KN, Rao PM, Rai MT, Sundaravadanan S. 1975. Copper glycinate therapy in repeat breeding cows. Ind $J$ Anim Res 7: 75-78.

18. Thomas JW, Moss S. 1951. The effect of orally administrated molybdenum on growth, spermatogenesis and testes histology of young dairy bulls. J Dairy Sci 34: 929-934.

19. Whitaker DA. 1982. A field to asses the effect of copper glycinate injections on fertility in dairy cows. Br Vet $J 138$ : 40-44.

20. Yunice AA, Lindeman RD. 1975. Effect of estrogen-progestogen administration on tissue cation concentrations in the rat. Endocrinology 97: 1263-1269.

\section{Revista Veterinaria obtuvo el máximo nivel de categorización del CAICYT-CONICET}

Tras el pertinente proceso de evaluación según criterios de calidad editorial, en setiembre de 2005 CAICYT-CONICET ha clasificado a nuestra publicación con Categoría 1 (nivel superior de excelencia), con lo cual pasa a integrar el Catálogo Latindex (folio 14022). La Dirección de Revista veterinaria agradece a quienes colaboraron para obtener tan importante distinción. Ver: http://www.latindex. unam.mx/busquedas/catalogotitulo.html 